\title{
Impact of User Mobility and Asymmetry on Multiuser Scheduler Performance
}

\author{
Pengcheng Zhan, Ramesh Annavajjala and A. Lee Swindlehurst
}

\begin{abstract}
In this paper, we are concerned with the performance of multiuser schedulers over an asymmetric network, where different users in the network have different average received signal-to-noise ratios (SNRs) and Doppler spreads. The throughput, in terms of the ergodic capacity of the channel, of a maximum-SNR scheduler (i.e., the scheduler that schedules the user with the largest instantaneous SNR) is investigated over Rayleigh fading channels with singleantenna base station and user terminals. Closed-form expressions are presented to quantify the degradation in maximumSNR scheduler performance due to lack of the knowledge of the CSI. For an asymmetric network with a small number of users, a new simple scheduler, which does not require instantaneous CSI, is proposed, and its optimality is discussed.
\end{abstract}

Keywords: Multiuser diversity, proportional fair, Doppler spread, fading decorrelation, channel-aware schedulers.

\section{INTRODUCTION}

In a single cell, with single-antenna base station (BS) and user terminals (UTs), scheduling the strongest user at every transmission time, for either the uplink (UL) or the downlink (DL), is known to be optimal in the Shannon capacity sense [1], [2]. By allowing only the UT with the strongest instantaneous received signal-tonoise ratio (SNR) to transmit (i.e., the so-called maximumSNR scheduler or, simply, max-SNR scheduler), the shared channel resource is used most efficiently, and results in maximum system throughput [3]. The resulting gain due to riding the peaks of the multiuser fading channel is termed multiuser diversity (MUD) gain [4], which, in a symmetric Rayleigh faded network (with equal average received SNRs for the UTs), grows doubly logarithmically with the number of UTs. However, when the network contains UTs with different average SNRs, maxSNR scheduler incurs unfairness, in the sense that the UT with the largest average SNR gets scheduled more often. The simplest scheduler that guarantees fairness among the UTs is a round-robin (RR) scheduler, which schedules the UTs in equal proportion, regardless of the instantaneous UT channel quality. A proportional-fair (PF) scheduler trades off the system throughput for fairness across UTs by scheduling the UTs based on the instantaneous channel

Pengcheng Zhan is with the Department of Electrical and Computer Engineering, Brigham Young University, Provo, UT 84602, USA. Email ezhan@byu.edu

Ramesh Annavajiala is with ArrayComm LLC, 2480 North First Street San Jose, CA, 95131, USA. Email: ramesh@arraycomm.com

A. Lee Swindlehurst is with the Department of Electrical Engineering and Computer Science, the University of California, Irvine, CA 92697 2625, USA. Email: swindle@uci.edu quality relative to the average channel quality over the time window of interest [4].

In this paper, our first goal is to understand the impact of dissimilar average SNRs and Doppler spreads on the max-SNR scheduler performance. Assuming DL transmission, accurate knowledge of channel state information (CSI) at the BS is crucial to exploit the benefits of MUD. The UT also should have access to good quality CSI to obtain sufficient decoder performance. Lack or inaccuracy of the CSI leads to a significant performance degradation [5], [6], whereas the overhead in maintaining CSI penalizes the system throughput. Typically, a max-SNR scheduler selects the UT with the strongest SNR based on the initial channel quality measurements (say, at time $t=0$ ), and allocates radio resources to the scheduled UT ${ }^{1}$ over a duration $T$ that is less than the coherence time $T_{c}$ of that user. Depending on the ability of the system to track the channel quality of the UTs, we study two scenarios in the paper. These are: $i$ ) Perfect CSI, and ii) Outdated CSI. For the first case, we assume the system has perfect CSI only for the active UT during the whole allocated time duration $T$ (i.e., tracking the channel quality of UT that is scheduled at $t=0$ ). In this case, there is a performance degradation due to the fact that the SNR of another user may increase to a value that is better than the scheduled user during the allocation window $T$. For the second case, after making a scheduling decision based on the channel quality at $t=0$, the BS makes no attempt to monitor the channel quality over the duration $T$. In this case, there is an additional loss due to decorrelated CSI. The practical significance of both cases $i$ ) and ii) is that they quantify the performance loss due to the lack of periodic channel quality feedback.

Our results for an asymmetric network with a small number of UTs, with different mobile speeds, show that the max-SNR scheduler may not necessarily perform better than a scheduler that does not have access to instantaneous CSI. The latter one is termed Statistically ChannelAware (SCA) scheduler, as it requires only the knowledge of the average received SNRs and the Doppler spreads of the UTs. We formulate a simple constrained optimization problem, which maximizes the average capacity of the proposed SCA scheduler, to allocate the time durations for the UTs. An algorithmic description of the SCA scheduler is also presented.

The rest of this paper is organized as follows. In Section II, we present the system model. A thorough

\footnotetext{
${ }^{1}$ The 'scheduled UT' is also termed 'active UT'.
} 
study of the performance of the max-SNR scheduler under different CSI assumptions is carried out in Section III. In Section IV, we expose the drawbacks of the max-SNR scheduler for a small network with high mobility users, and propose our SCA scheduler. We conclude our work in Section V.

\section{SYSTEM MODEL}

We consider a single cell with a small group of $K$ independently faded asymmetric users. Assuming singleantenna BS and UTs, we focus on DL (i.e., BS to UT) transmission. At time $t$, for UT $j, 1 \leq j \leq K$, the instantaneous received SNR is denoted by $\gamma_{j}(t)$, whereas the corresponding statistical average is denoted by $\overline{\gamma_{j}(t)}$. We also assume that the users move at different speeds, and the resulting Doppler spread for the $j$ th UT is given by $f_{d}^{j}=\left(v_{j} / c\right) f_{c}$, where $f_{c}$ is the carrier frequency, $c$ is the speed of light, and $v_{j}$ is the speed of UT $j$. The complexvalued time-varying channel gain from BS to UT $j$, at time $t$, is denoted by $h_{j}(t)$, which is assumed to be a zeromean complex-Gaussian (ZMCG) random variable (r.v ${ }^{2}$ with second moment $\mathrm{E}\left[\left|h_{j}(t)\right|^{2}\right]=\Omega_{h_{j}}(t)$. With this, the received signal at UT $j$ is:

$$
y_{j}(t)=h_{j}(t) x(t)+n_{j}(t),
$$

where $x(t)$ is the transmitted signal with $\mathrm{E}\left(|x(t)|^{2}\right)=$ $E_{s}(t)$ denoting the average transmit power, and $n_{j}(t) \sim$ $\mathcal{C N}\left(0, \zeta_{j}^{2}\right)$. With this, we have $\gamma_{j}(t)=\left|h_{j}(t)\right|^{2} E_{s}(t) / \zeta_{j}^{2}$ and $\overline{\gamma_{j}(t)}=\Omega_{h_{j}}(t) E_{s}(t) / \zeta_{j}^{2}$.

Due to the users' mobility, the channel for UT $j$ changes for values of $t$ greater than the coherence time $T_{c}^{j}$. Since $h_{j}(t)$ is $Z \mathrm{MCG}, h_{j}(t)$ and $h_{0}(t)$ are related as [7]:

$$
h_{j}(t)=\rho_{j}(t) \sqrt{\frac{\Omega_{h_{j}}(t)}{\Omega_{h_{j}}(0)}} h_{j}(0)+\sqrt{\left(1-\left|\rho_{j}(t)\right|^{2}\right\rangle \Omega_{h_{j}}(t)} v_{j}(t),
$$

where $v_{j}(t) \sim \mathcal{C N}(0,1)$. Assuming the Jakes fading correlation model [8] for each user, we write $\rho_{j}(t)=\mathcal{J}_{0}\left(2 \pi f_{d}^{j} t\right)$, where $\mathcal{J}_{0}(\cdot)$ is the zero-th order Bessel function of the first kind [9]. We denote by $T$ the time slot duration for this group of users (a BS can serve different groups of users), within which scheduler can make the decisions about which user to schedule, how much time to schedule it and so on. We further assume that $T \leq \min _{1 \leq j \leq K}\left(T_{c}^{j}\right)$.

\section{MAX-SNR SCHEDULER}

As mentioned before, the system performance is a function of the quality of CSI at both the BS and UTs. The UTs usually estimate the CSI by extracting the pilots inserted in the transmitted data. The BS can obtain the CSI either by estimating the channel through the reverse link (assuming channel reciprocity), or via the feedback from UTs through a control channel. Keeping track of the CSI will incur a a penalty in system throughput. Depending on the ability to track the CSI, this section addresses the max-SNR scheduler performance under different scenarios.

\footnotetext{
${ }^{2}$ For simplicity, we denote $\mathrm{X} \sim \mathcal{C} \mathcal{N}\left(m_{t} \sigma^{2}\right)$ to indicate that $\mathrm{X}$ is a Complex Gaussian r.v with mean $m$ and variance $\sigma^{2}$.
}

\section{A. Ideal Performance}

Under this assumption that the BS has perfect CSI of all the UTs during $0 \leq t \leq T$, the pdf of the maximum of the instantaneous SNR, at any $t \in[0, T]$, can be written as:

$$
p_{\gamma_{\max }, d}(x)=\sum_{j=1}^{K} \sum_{j_{j}=0}^{1} \sum_{p=1}^{K} \frac{l_{p}(-1)^{\sum_{j=1}^{K} l_{j}+1} e^{-x \sum_{q=1}^{K} \frac{l_{q}}{\gamma_{q}(l)}}}{\gamma_{p}(t)} .
$$

The Shannon capacity of this scheduler is given by:

$$
\begin{aligned}
C(t) & =\int_{0}^{\infty} \log _{2}(1+x) p_{\gamma_{\max }, l}(x) d x \\
& =\log _{2}(e) \sum_{j=1}^{K} \sum_{j=0}^{1}(-1)^{1+\sum_{j=1}^{K} l_{j}} e^{\left(\sum_{j=1}^{K} \frac{l_{j}}{\gamma_{j}(t)}\right)} \varepsilon_{1}\left(\sum_{j=1}^{K} \frac{l_{j}}{\gamma_{j}(t)}\right),
\end{aligned}
$$

where $\mathcal{E}_{1}(x)=\int_{x}^{\infty} 1 / t \cdot e^{-t} \cdot d t$ is the exponential integral function [9]. When the transmission power and the average channel gains are time-invariant (i.e., no Doppler), $\overline{\gamma_{j}(t)}$ will remain time-invariant. In this scenario, the Shannon capacity in (4) remains constant over the period $[0, T]$.

\section{B. Channel Tracking of Scheduled User}

Due to high CSI requirements, the scheduler described in Section III-A is impractical. Here, we investigate the case when the scheduler has the CSI for all the users only at time $t=0$, based on which the initial scheduling decision is made for the duration $[0, T]$. In this period, only the active UT's channel is tracked at the UT side for the purpose of decoding the message, and is assumed to be perfectly known. Compared to the ideal scheduler, this system takes a performance hit due to not monitoring the channel quality of other UTs beyond $t=0$. In this subsection, we quantify this loss.

From (2), conditioned on $h_{j}(0)$, it is easy to see that $\gamma_{j}(t)=\left|h_{j}(t)\right|^{2} E_{s}(t) / \zeta_{j}^{2}$ is a non-central Chi-square r.v with the parameters [10]:

$$
s_{j}^{2}(t)=\left|\rho_{j}(t)\right|^{2}\left|h_{j}(0)\right|^{\frac{\Omega_{h_{j}}}{\Omega_{h_{j}}(0)}} \frac{E_{s}(t)}{\zeta_{j}^{2}}=\frac{\left|\rho_{j}(t)\right|^{2} \overline{\gamma_{j}(t)}}{\Omega_{h_{j}}(0)}\left|h_{j}(0)\right|^{2}
$$

and

$$
2 \sigma_{j}^{2}(t)=\left\langle 1-\left|\rho_{j}(t)\right|^{2}\right\rangle \Omega_{h_{j}}(t) \frac{E_{s}(t)}{\zeta_{j}^{2}}=\left\langle 1-\left|\rho_{j}(t)\right|^{2}\right\rangle \overline{\gamma_{j}(t)} .
$$

Therefore, the cumulative distribution function (CDF) of $\gamma_{j}(t)$ conditioned on $h_{j}(0)$ is [10], [11]:

$$
P\left(\gamma_{j}(t) \leq x \mid h_{j}(0)\right)=1-\mathcal{Q}_{1}\left(\frac{s_{j}(t)}{\sigma_{j}(t)}, \frac{\sqrt{x}}{\sigma_{j}(t)}\right),
$$

where $\mathcal{Q}_{1}(a, b)$ is the first-order Marcum-Q function [10]. It can be easily shown that the pdf of the maximum SNR, with UT $j$ being the strongest user at time $t=0$ is:

$$
\begin{aligned}
p_{\gamma_{j} j}(x, j, t=0) & =\frac{e^{-\frac{x}{\gamma_{j}(0)}}}{\overline{\gamma_{j}(0)}} \prod_{i=1, i \neq j}^{K}\left(1-e^{-\frac{x}{\gamma_{i}(0)}}\right) \\
& =\sum_{i=1, i \neq j}^{K} \sum_{i_{i}=0}^{1}(-1)^{\sum_{i=1, i \neq j}^{K}} \frac{e^{-\left(\sum_{i=1, i \neq j}^{K} \frac{l_{i}}{\gamma_{i}(0)}+\frac{1}{\gamma_{j}(0)}\right) x}}{\overline{\gamma_{j}(0)}} .
\end{aligned}
$$

Upon defining

$$
\begin{aligned}
F(\alpha, k) & \triangleq \frac{e^{\alpha}}{k !} \sum_{j=0}^{K}(-1)^{j}\left(\begin{array}{c}
k \\
j
\end{array}\right) \alpha^{j} \int_{\alpha}^{\infty} e^{-i} t^{k-j-1} d t \\
& =e^{\alpha} \sum_{j=0}^{K}(-1)^{j}\left(\begin{array}{c}
k \\
j
\end{array}\right) \alpha^{j} \frac{G(\alpha, k-j)}{k(k-1) \cdots(k-j)},
\end{aligned}
$$


where $G(\alpha, k)=\frac{1}{(k-1) !} \int_{\alpha}^{\infty} e^{-t} t^{k-1} d t$ is the incomplete gamma function [9], the Shannon capacity of a max-SNR scheduler that tracks the channel of the active UT for the whole time slot $T$ can be derived as:

$$
\begin{aligned}
& C(t)=\sum_{j=1}^{K} \int_{0}^{\infty} C_{j \mid h_{j}(0)}(t) p_{\eta_{j}(0), j}(x, j, t=0) d x \\
& =\sum_{j=1}^{K} \sum_{i=1, i \neq j}^{K} \sum_{l_{i}=0}^{1} \sum_{n=0}^{\infty} \sum_{k=0}^{n} \frac{\log _{2}(e)}{\overline{\gamma_{j}}(0)} \frac{\left(b_{j}(t)\right)^{n}(-1)^{\sum_{i=1, i \neq j}^{K} l_{i} F\left(\alpha_{j}(t), k\right)}}{\left(\sum_{i=1, i \neq j}^{K} \frac{l_{i}}{\gamma_{i}(0)}+\frac{1}{\gamma_{j}(0)}+b_{j}(t)\right)^{n+1}},
\end{aligned}
$$

where $\alpha_{j}(t)=\frac{1}{\left(1-\left|\rho_{j}(t)\right|^{2}\right) \overline{\gamma_{j}(t)}}$ and $b_{j}(t)=\frac{\left|\rho_{j}(t)\right|^{2}}{\left(1-\left|\rho_{j}(t)\right|^{2}\right) \overline{\gamma_{j}(0)}}$. Details of (9) can be found in [12].

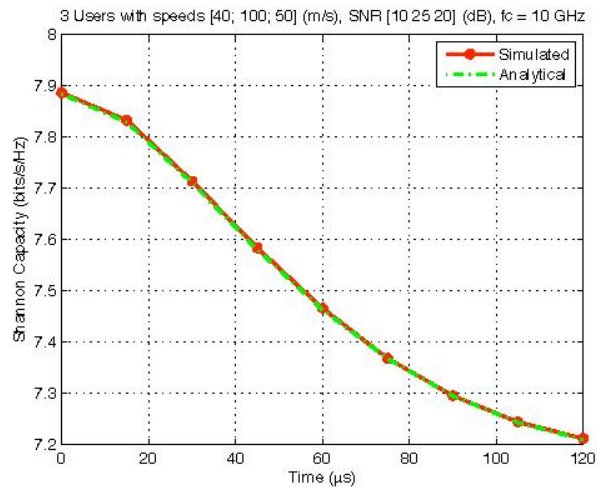

Fig. 1. Shannon capacity of a max-SNR scheduler. Only the scheduled user at $t=0$ has access to perfect CSI over $t \in[0, T]$.

A Monte-Carlo simulation is carried out to simulate the Shannon capacity of the max-SNR scheduler over a network with 3 asymmetric users. At time $t=0$, each user's channel is generated randomly, and the strongest user is selected according to the users' instantaneous SNR at this time. After the user is selected, random samples are generated to evolve the channel according to (2). The Shannon capacity is measured by averaging over a large number of channel realizations. For simplicity, the second order statistics of each user's channel is assumed to be constant (i.e., $\Omega_{h_{j}}(t)=C, t \in[0, T]$ ), and each user's transmit power and noise variance are also set to be constant during the scheduled time slot. The carrier frequency is set to $f_{c}=10 \mathrm{GHz}$, the average SNRs of the users is set to $[15,25,20] \mathrm{dB}$, the Doppler speed is set to $[40,100,50] \mathrm{m} / \mathrm{s}$, and the time slot duration $T$ is set to be $120 \mu \mathrm{s}$. The same set of parameters will be used throughput the paper. As can be seen in Fig. 1, the simulated results agree very well with the analysis in (9). The performance loss can be quantified as the difference between (4) and (9).

\section{Impact of Channel Tracking Inability}

When the channel feedback frequency is severely constrained, the UTs provide only the initial CSI to the BS scheduler. Once a scheduling decision is made, during the scheduling period the BS will make no further attempts to track the channel of any of the UTs. More importantly, at the UT side, the receiver doesn't track its own channel.
The initially obtained CSI is used throughout its scheduled time to decode the message. Clearly, the required CSI load in this case is significantly less in comparison with the approaches in Sections III-A and III-B. However, not tracking the channel at all, after $t=0$, leads to further performance degradation over the one described in Section III-B.

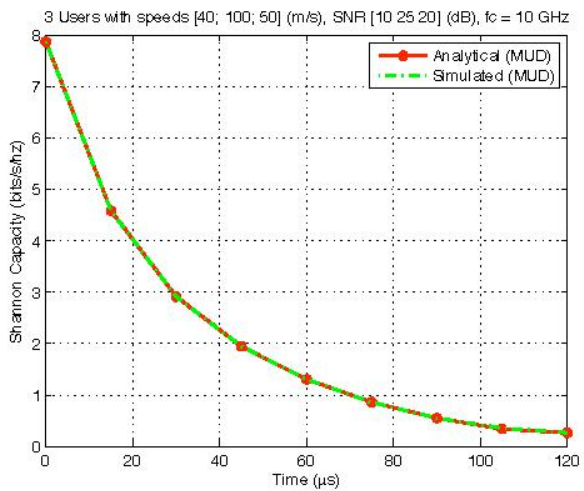

Fig. 2. Shannon capacity of the max-SNR scheduler. Channel knowledge is known for the selected user only at the scheduling time.

Using (2) in (1), the instantaneous SNR of UT $j$ given $h_{j}(0)$ is:

$$
\begin{aligned}
\eta_{j}(t) & \triangleq \frac{\left|\rho_{j}(t)\right|^{2}\left|h_{j}(0)\right|^{2} \frac{\Omega_{h_{j}}(t)}{\Omega_{h_{j}}(0)} E_{s}(t)}{\left(1-\left|\rho_{j}(t)\right|^{2}\right\rangle \Omega_{h_{j}}(t) E_{s}(t)+\zeta_{j}^{2}} \\
& =\frac{\left|\rho_{j}(t)\right|^{2}\left|h_{j}(0)\right|^{2} E_{s}(t)}{\Omega_{h_{j}}(0)\left[\left(1-\left|\rho_{j}(t)\right|^{2}\right\rangle E_{s}(t)+\frac{\zeta_{j}^{2}}{\Omega_{h_{j}}(t)}\right]}=\phi_{j}(t) \eta_{j}(0)
\end{aligned}
$$

where

$$
\begin{aligned}
\phi_{j}(t) & \triangleq \frac{\left|\rho_{j}(t)\right|^{2} E_{s}(t)}{E_{s}(0) / \zeta_{j}^{2} \Omega_{h_{j}}(0)\left[\left(1-\left|\rho_{j}(t)\right|^{2}\right) E_{s}(t)+\frac{\zeta_{j}^{2}}{\Omega_{h_{j}}(l)}\right]} \\
& =\frac{\left|\rho_{j}(t)\right|^{2} \overline{\gamma_{j}(t)}}{\overline{\gamma_{j}(0)\left[\left(1-\left|\rho_{j}(t)\right|^{2}\right) \overline{\gamma_{j}(t)}+1\right]} .}
\end{aligned}
$$

Averaging over $\eta_{j}(t)$ and the users, we obtain the Shannon capacity in the absence of channel tracking as [12]:

$$
\begin{aligned}
& C(t)=E_{j}\left(E_{y_{j}(l)}\left(\log _{2}\left(1+\eta_{j}(t)\right)\right)\right) \\
& =\sum_{j=1}^{K} \frac{\log _{2} e}{\overline{\gamma_{j}(0)}} \sum_{i=1, i \neq j}^{K} \sum_{l_{i}=0}^{1} \frac{(-1)^{\sum_{i=1, i \neq j}^{K} l_{i}}}{\sum_{i=1, i \neq j}^{K} \frac{l_{i}}{\gamma_{i}(0)}+\frac{1}{\gamma_{j}(0)}} e^{\frac{\sum_{i=1, i \neq j}^{K} \frac{l_{i}}{\gamma_{i}(0)}+\frac{1}{\gamma_{j}(0)}}{\phi_{j}(t)}} \mathcal{E}_{1}\left(\frac{\sum_{i=1, i \neq j}^{K} \frac{4}{\gamma_{i}(0)}+\frac{1}{\gamma_{j}(0)}}{\phi_{j}(t)}\right) .
\end{aligned}
$$

We perform a Monte-Carlo simulation for this scheduler. The result shown in Fig. 2 verifies the accuracy of the analysis. We also observe from Fig. 2 that the loss in capacity is significant when the scheduler does not keep track of the user's channel (6 bits/s/Hz loss in Fig. 2 as compared to 0.95 bits $/ \mathrm{s} / \mathrm{Hz}$ loss in Fig. 1 at the end of the scheduling time $T$ ). The difference between (9) and (12) characterizes additional loss due to UT's not tracking its own channel. 


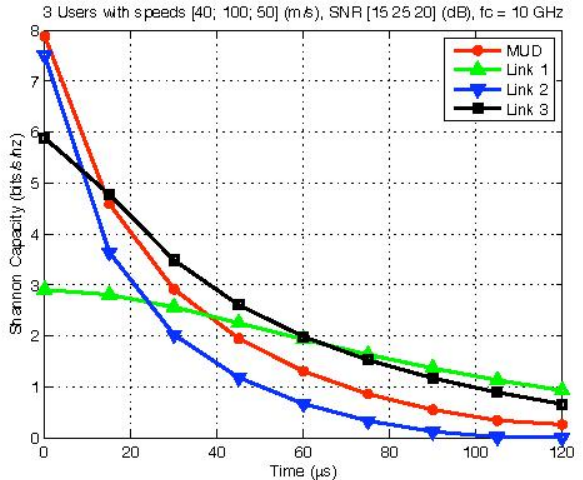

Fig. 3. Shannon capacity of multiuser schedulers. Channel knowledge is known for the selected user only at the scheduling time.

\section{SCA SCHEDULER}

As seen from Fig. 2, when the strongest user (in terms of average SNR) in the network has a large Doppler spread, the Shannon capacity of the max-SNR scheduler decays quickly with time. We conjecture that for a small network, where the number of asymmetric users is not large, scheduling the strongest user without tracking its channel for a period of time may not provide better throughput than simply selecting a user with a similar average SNR, but with a smaller Doppler spread. This is attributed to the observation that high mobility will outdate the channel knowledge very quickly. Under these conditions, Doppler spread as well as average SNR should be key factors to be considered for making scheduling decisions. Here, we consider a simple statistically channelaware scheduler that requires only the average SNRs and the Doppler spreads as inputs. As motivation, consider a scheduler that selects a user, say $\dot{j}$, and allocates time slot $T$ to it regardless of how good its channel is when compared to the other of users. The selected user's Shannon capacity is given by [12]:

$$
\begin{aligned}
C_{j}(t) & =\mathrm{E}\left(\log _{2}\left(1+\phi_{j}(t) \eta_{j}(0)\right)\right) \\
& =\log _{2} e \times e^{\frac{\left(1-\left|\rho_{j}(t)\right|^{2} \overline{\gamma_{j}(t)}+1\right.}{\left|\rho_{j}(t)\right|^{2} \gamma_{j}(t)}} \varepsilon_{1}\left(\frac{\left(1-\left|\rho_{j}(t)\right|^{2}\right) \overline{\gamma_{j}(t)}+1}{\left|\rho_{j}(t)\right|^{2} \overline{\gamma_{j}(t)}}\right) .
\end{aligned}
$$

In Fig. 3, we plot the Shannon capacity of the maxSNR scheduler and this simple scheduler according to (12) and (13), respectively. From Fig. 3, we conclude that: a) selecting the strongest at user $t=0$, and scheduling it for $T=120 \mu \mathrm{s}$ without tracking its channel, is not as good as simply selecting user ' 3 ' for the whole time duration, and b) the gain of MUD over selecting the user with the largest average SNR (user ' 2 ') is marginal. The authors attribute these observations to two reasons: 1 ) the number of the users in the network is not large, hence the possible MUD gain is not large to start with, and 2) due to the asymmetric network setup, with high probability, the max-SNR scheduler selects user ' 2 ' who has the strongest average SNR. However, due to high Doppler spread, user ' 2 ' channel de-correlates much quicker than the rest of the users. Therefore, in a small network with some high mobility users, MUD without the CSI for the whole scheduling time may lead to suboptimal performance.

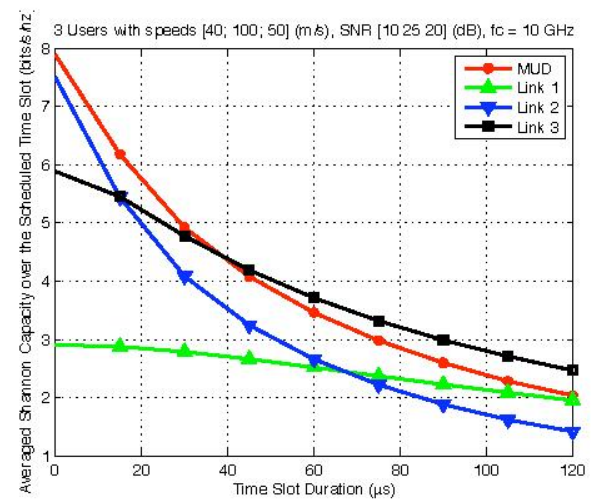

Fig. 4. Average Shannon capacity of max-SNR scheduler, and the capacity of a given user link.

To determine whether to use a max-SNR scheduler or a SCA scheduler, one reasonable approach would be to average (12) and (13) over $[0, T]$, and use them as the metrics for user selection. Fig. 4 plots such metrics mentioned above as a function of scheduled time slot duration. It is clear from Fig. 4 that there are crossover points for different curves in the figure. Therefore, besides the users' average SNRs and Doppler spreads, the slot duration is also an important parameter to determine the optimal scheduler type (max-SNR versus SCA), and user selection. When $T=120 \mu$, user 3 is the optimal user to be scheduled. However, it neither corresponds to the user with the largest average SNR nor corresponds to the user with the smallest Doppler spread. When $T \lesssim 40 \mu s$, the max-SNR scheduler that extracts the MUD gain would be the right choice. For the rest of this paper, we dedicate our attention to the SCA scheduler when the MUD gain is not of much concern. However, which user to schedule, and for how much time, will be the focus of the rest of this section.

\section{A. A Sufficient Condition for Optimal User Selection}

In this section, we study a sufficient condition for an SCA scheduler under which scheduling a specific user for the whole period of time duration $T$ is optimal. We assume the selected user only estimates its own channel at time $t=0$ at the receiver side for the purpose of decoding the message. At the scheduler side, no instantaneous CSI is required to determine the prospective user. Only average SNR and Doppler spread information is needed to make the scheduling decision. As explained in the last paragraph, the optimal scheduling strategy is a function of $T$. To answer the question Which user is the optimal user to be scheduled, integration over $[0, T]$ is involved. When the scheduled time duration $T$ is smaller than the minimum of all the intersecting points at which the capacity curve (averaged over $[0, T]$, i.e. Fig. 4 ) of the user with the largest average SNR crosses the remaining curves, the user with the largest average SNR is the optimal user to schedule. And in this case, an SCA scheduler bases its decision only on users' average SNR. For example, in Fig. 4 , if $T$ is less 
than the intersecting point of the blue curve and the black curve, i.e. $T \lesssim 15 \mu$, scheduling user ' 2 ' for the whole $T$ is optimal.

To determine the intersecting points in Fig. 4 is hard, because integration is involved. It is obvious that the intersecting point in Fig. 3 always lower bounds the desired one. Therefore, we propose to determine the intersecting point in Fig. 3. When $T<T_{c}^{j}$ and if $2 \pi \cdot f_{d}^{j} \cdot T<4$, from [13], we can use the polynomial approximation of Bessel functions: $\mathcal{J}_{0}(x) \approx \sum_{m=0}^{n} C_{n m} x^{2 m}$, with $n=2$ and $C_{n m}=\frac{(-1)^{m} n^{1-2 m}(n+m-1) !}{2^{2 m}(n-m) !(m !)^{2}}$. Since each term in (13) has the form $e^{x} \mathcal{E}_{1}(x)$, we can prove that the latter is monotonically decreasing in $x$ [12]. It can be shown that the following holds true when the capacity curves of users $i$ and $j$ intersect:

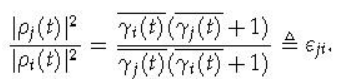

Using polynomial approximation for $\rho_{j}(t)=\mathcal{J}_{0}\left(2 \pi f_{d}^{j} T\right)$ :

$$
\left(\frac{\sum_{m=0}^{n} C_{n m} x_{j}^{2 m}}{\sum_{m=0}^{n} C_{n m} x_{i}^{2 m}}\right)^{2}=\varepsilon_{j i r}
$$

where $x_{i}=2 \pi f_{d}^{i} T$. Upon setting $x_{j} / x_{i}=f_{d}^{j} / f_{d}^{i} \triangleq k_{j i}$, an approximation to (15), with $n=2$, is given by

$$
\sum_{m=1}^{2} C_{2 m}\left(k_{j i}^{2 m}-\sqrt{\epsilon_{i j}}\right) x_{i}^{2 m}+C_{20}\left(1-\sqrt{\epsilon_{j i}}\right)=0 .
$$

In (16), we determine the smaller positive root $x_{i}$, which can be divided by $2 \pi f_{d}^{i}$ to obtain $T_{i, j}, i \neq j$. If user $v$ is the one with the largest average SNR, we call $T_{B}=\min \left(T_{v, 1}, \cdots, T_{v, v-1}, T_{v, v+1}, \cdots T_{v, K}\right)$. If $T \leq T_{B}$, it is guaranteed that scheduling user $v$ for the whole period of $T$ will be optimal.

\section{B. Time Resource Allocation for an SCA Scheduler}

This section studies a different problem, i.e. when a scheduler is constrained to have only access to all users' long term statistics (average SNR and Doppler spread), how to intelligently allocate time resource among various users to maximize the system throughput. Mathematically, $K$ asymmetric users are assumed in the network with different Doppler spread. Total amount of time $T$ is assumed to be dedicated to serving these users. As discussed in IV-A, if $T \leq T_{B}$, scheduling the user with the strongest average $\mathrm{SNR}$ is optimal. Otherwise, it may be optimal in the Shannon capacity sense to allocate the total time resource to various users. The optimal solution for this scenario can be obtained by formulating following optimization problem:

$$
\begin{gathered}
{\left[T_{1}, \cdots, T_{K}\right]=\underset{\left[x_{1}, \cdots, x_{K}\right]}{\arg \max } f\left(\left[x_{1}, \cdots, x_{K}\right]\right)} \\
\text { s.t. } \sum_{j=1}^{K} x_{j}=T \text {, and } x_{j} \geq 0 \text {, for } 1 \leq j \leq K,
\end{gathered}
$$

where

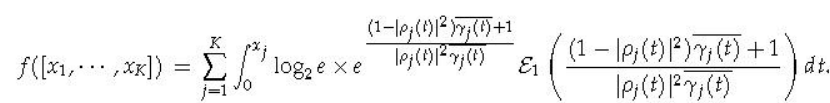

The gradient of the above cost function can be easily evaluated by plugging $x_{j}$ 's into (13):

$$
\frac{\partial f}{\partial x_{j}}=\log _{2} e \cdot e^{\frac{\left(1-\left|\rho_{j}\left(x_{j}\right)\right|^{2}\right) \overline{\gamma_{j}\left(x_{j}\right)}+1}{\left|\rho_{j}\left(x_{j}\right)\right|^{\gamma} \gamma_{j}\left(x_{j}\right)}} \varepsilon_{1}\left(\frac{\left(1-\left|\rho_{j}\left(x_{j}\right)\right|^{2}\right) \overline{\gamma_{j}\left(x_{j}\right)}+1}{\left|\rho_{j}\left(x_{j}\right)\right|^{2} \gamma_{j}\left(x_{j}\right)}\right) .
$$

Any gradient based numerical optimization technique can be used to find the optimal time allocation for each user (i.e., $\left[T_{1}, \cdots, T_{K}\right]$ ). In our simulation, the total amount of time $T$ is set to be $120 \mu \mathrm{s}$. The averaged Shannon capacity of this optimal scheduler is found to be 3.6023 bits/s/Hz. User " 1 " is scheduled with $42.17 \mu$ s, user " 2 " is scheduled with $26.31 \mu \mathrm{s}$, and user " 3 " is scheduled with the rest $51.51 \mu \mathrm{s}$. The method of obtaining this solution guarantees the optimality of the time resource allocation.

\section{CONCLUSION}

In this paper, we investigated the impact of user mobility and asymmetry on the multiuser scheduler performance. Closed-form expressions were derived for the max-SNR scheduler performance under various assumptions on the level of CSI. Our simulations showed that channel-aware multiuser scheduling is not always optimal for a small network with a large Doppler spread when the slot duration is inappropriate and the user is not tracking its own channel for decoding. Over such networks, we proposed a simple SCA scheduler that achieves improvements to the system throughput.

\section{REFERENCES}

[1] R. Knopp and P. Humblet, "Information capacity and power control in single-cell multiuser communications," in IEEE International Conference on Communications, vol. 53, June 1995, pp. 331-335.

[2] D. Tse, "Optimal power allocation over parallel gaussian broadcast channels," in Proceedings of IEEE International Symposium on Information Theory, 1997, p. 27.

[3] P. Viswanath, D. N. C. Tse, and R. Laroia, "Opportunistic beamforming using dumb antennas," in IEEE Transactions on Information Theory, vol. 48, June 2002, pp. 1277-1294.

[4] D. Tse and P. Viswanath, Fundamentals of Wireless Communication. Cambridge University Press, 2005.

[5] D. Piazza and L. Milstein, "Multiuser diversity-mobility tradeoff: Modeling and performance analysis of a proportional fair scheduling," in IEEE Global Telecommunications Conference, vol. 1, 2002, pp. 906-910.

[6] Q. Ma and C. Tepedelenlioglu, "Practical multiuser diversity with outdated channel feedback," in IEEE Transactions on Vehicular Technology, vol. 54, 2005, pp. 1334-1345.

[7] T. Anderson, An Introduction to Multivariate Statistical Analysis. Wiley Series in Probability and Statistics, 2003.

[8] W. Jakes, Microwave Mobile Communications. Wiley, 1974.

[9] M. Abramowitz and I. A. Stegun, Handbook of Mathematical Functions. Applied Mathematics Series 55. New York: National Bureau of Standard, 1964

[10] J. G. Proakis, Digital Communications. McGraw Hill, 2001.

[11] M. Simon and M. Alouini, Digital Communication over Fading Channels. Wiley, 2004

[12] P. Zhan, "Optimizing wireless network throughput: Methods and applications," in PhD Dissertation, Brigham Young University, 2007.

[13] R. Millane and J. Eads, "Polynomial approximations to bessel functions," in IEEE Transactions on Antennas and Propagation, vol. 51, Jun. 2003, pp. $1398-1400$. 